\title{
PROTECTION AGAINST SHIGELLOSIS CAUSED BY SHIGELLA DYSENTERIAE SEROTYPE 4 IN GUINEA PIGS USING ESCHERICHIA ALBERTII DM104 AS A LIVE VACCINE CANDIDATE STRAIN
}

\author{
FATEMA MONI CHOWDHURY ${ }^{1,2,3}$, MOHAMMED ZIAUR RAHMAN ${ }^{1,2,4}$,

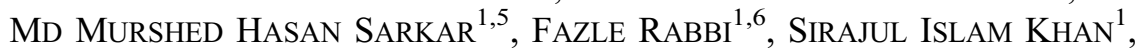 \\ CHOWDHURY RAFIQUL AHSAN ${ }^{1 *}$ and NILS-KÅRE BIRKELAND ${ }^{2}$ \\ ${ }^{1}$ Department of Microbiology, University of Dhaka, Dhaka, Bangladesh \\ ${ }^{2}$ Department of Biology, University of Bergen, Bergen, Norway \\ ${ }^{3}$ Present address: Department of Microbiology, Jagannath University, 9-10 Chittaranjan \\ Road, Dhaka 1100, Bangladesh \\ ${ }^{4}$ Present address: International Centre for Diarrheal Disease Research, Bangladesh \\ (icddr,b), GPO Box 128, Dhaka 1000, Bangladesh \\ ${ }^{5}$ Present address: Department of Immunology, Graduate School of Medicine, Chiba \\ University, 1-8-1 Inohana, Chuo-ku, Chiba-city, Chiba 260-8670, Japan \\ ${ }^{6}$ Present address: Microbiology Program, Department of Mathematics and Natural \\ Sciences, BRAC University, 66 Mohakhali, Dhaka 1212, Bangladesh
}

(Received: 25 May 2016; accepted: 27 October 2016)

Recently, we reported the induction of protective immunity by environmental Escherichia albertii strain DM104 against Shigella dysenteriae in guinea pig model. In this study, we assessed three different immunization routes, such as intranasal, oral, and intrarectal routes, and revealed differences in immune responses by measuring both the serum IgG and mucosal IgA antibody titers. Protective efficacy of different routes of immunization was also determined by challenging immunized guinea pigs against live $S$. dysenteriae. It was found that intranasal immunization showed promising results in terms of antibody response and protective efficacy. All these results reconfirm our previous findings and additionally point out that the intranasal immunization of the environmental E. albertii strain DM104 in guinea pig model can be a better live vaccine candidate against shigellosis.

Keywords: live, Shigella, shigellosis, vaccine

\footnotetext{
*Corresponding author; E-mail: crahsan@du.ac.bd
} 


\section{Introduction}

Shigella species (spp.) are the causative agents of acute diarrheal disease, shigellosis, with symptoms including abdominal cramps, watery or bloody diarrhea, and fever. Recent estimates represent diarrhea as the third-leading cause of infant mortality worldwide [1], and those most affected by shigellosis are children below the age of 5 [2-4]. There are four different Shigella spp. causing mild to severe diarrhea and attempts have already been taken under consideration for vaccine development [5, 6]. Among these, Shigella flexneri and Shigella dysenteriae are more prevalent in developing countries, whereas Shigella sonnei appears in regions with advanced sanitation standards. Ideally, a successful vaccine against Shigella is expected to be highly immunogenic without adverse side effects. While both liveattenuated Shigella strains and parenteral conjugate vaccine candidates have shown different degrees of success in human volunteer and non-human primate studies, licensed vaccines against shigellosis are yet to be available [7].

In recent years, our group has isolated a number of Shigella-like bacteria from freshwater environments in Bangladesh that serologically cross-reacted with different Shigella spp. serotypes [8-10]. One of these strains included an environmental isolate of Escherichia albertii (strain DM104), cross-reacting with $S$. dysenteriae type 4 [10]. DM104 strain was isolated from the Buriganga river in Dhaka, Bangladesh [11]. The strain was phylogenetically identified as E. albertii and showed a similar lipopolysaccharide (LPS) gel banding profile to that of $S$. dysenteriae type 4 [10]. Recently, it was also demonstrated that the DM104 isolate was non-invasive, did not produce any entero- or cytotoxins, and showed negative results in the mouse lethal activity assay [12]. The non-pathogenic DM104 strain gave, however, a high protective efficacy as an ocularly administered vaccine in the guinea pig eye model against $S$. dysenteriae type 4 challenge. It also induced a high titer of serum IgG against $S$. dysenteriae type 4 whole cell lysate (WCL) and LPS. On the whole, DM104 could be a good choice as a live vaccine candidate against shigellosis. In this study, we compared three different routes for immunization with the DM104 vaccine in guinea pigs to understand the better route of administration of the DM104 vaccine and its immunogenicity and protective efficacy in animal model.

\section{Materials and Methods}

\section{Bacterial strains}

E. albertii strain DM104 [10] and $S$. dysenteriae type 4 were obtained from the stock culture of the Department of Microbiology, University of Dhaka, Dhaka, Bangladesh. 
Animals

Male Hartley guinea pigs (body weight: 200-250 g) were maintained in the Department of Microbiology, University of Dhaka, and all experiments using animals were undertaken following the ethical issues set by the Faculty of Biological Sciences, University of Dhaka.

\section{Immunization}

Intranasal and oral immunizations. Guinea pigs were divided into four groups with 10 guinea pigs in each. Two groups were immunized with live bacteria through the intranasal and oral routes [13], and other two groups were used as negative control for each immunization scheme. An overnight bacterial culture in Brain Heart Infusion Broth was harvested, and the bacterial pellet was suspended in phosphate-buffered saline (PBS) at a concentration of approximately $10^{9} \mathrm{CFU}$ (colony-forming unit)/ml by comparing with McFarland standards. Guinea pigs were anesthetized with diethyl ether and immunized with the live DM104 bacteria either intranasally or orally on days 0,14 , and 28 . For intranasal immunization, a suspension of the live DM104 bacteria was applied drop wise to both the nostrils $(50 \mu \mathrm{l}$ each, $100 \mu \mathrm{l}$ total) of the guinea pigs. For oral immunization, $500 \mu \mathrm{l}$ of live DM104 bacteria was administered through an orogastric tube (no. 6) followed by a dose of $500 \mu$ of $1.4 \%$ sodium bicarbonate to neutralize the gastric acidity. Guinea pigs immunized with PBS only were considered as a negative control group.

Intrarectal immunization. A total of eight guinea pigs were anesthetized before being immunized through the intrarectal route with $5 \times 10^{8} \mathrm{CFU}$ of DM104 cells in $100 \mu \mathrm{l}$ PBS on days 0 and 14 [14]. Another group of eight guinea pigs immunized with PBS only was used as the negative control group.

\section{Collection of serum and other body fluids}

Two guinea pigs from each intranasally and orally immunized and control groups were used for collection of blood, eye wash, and intestinal lavage fluid, one day before the challenge experiment. Sera were separated from the blood and stored in aliquots at $-20{ }^{\circ} \mathrm{C}$ until used. Eye wash was collected from the guinea pigs by instilling $30 \mu \mathrm{l}$ of PBS in each eye. Intestinal lavage fluid fluids were collected from the guinea pigs as described by Orr et al. [15]. In brief, 20-25 cm of the small intestine, mainly the jejunum portion, was removed, and $0.5 \mathrm{ml}$ of PBS containing $0.1 \%$ bovine serum albumin, $50 \mathrm{mM}$ ethylenediaminetetraacetic acid, 
and $0.1 \mathrm{mg}$ of soybean trypsin inhibitor per $\mathrm{ml}$ was passed through it and intestinal fluid was collected. Phenylmethylsulfonyl fluoride was then added to the fluid ( $1 \mathrm{mM}$, final concentration), followed by vigorous vortexing and centrifugation at $1,000 \times g$ for $20 \mathrm{~min}$ to remove cells and debris. After supernatant collection, sodium azide $(0.1 \%$, final concentration) was added and the preparation was stored at $-20{ }^{\circ} \mathrm{C}$ until used. One week after the second immunization dose through intrarectal route, two of the guinea pigs from each group were sacrificed for blood sera collection.

\section{Measurement of antibody titers}

Enzyme-linked immunosorbent assay (ELISA) was performed to quantify the serum IgG and IgA and mucosal IgA against WCL of DM104 and $S$. dysenteriae type 4 [16]. Wells were precoated with DM104 and $S$. dysenteriae type $4 \mathrm{WCL}(1 \mu \mathrm{g} / \mathrm{well})$ and incubated with serially diluted guinea pig sera as the primary antibody. IgG and IgA antibody titers were determined using peroxidaselabeled goat anti-guinea pig IgG (Sigma, USA) and IgA (Innovative Research, USA). Endpoint titers were determined for each serum by taking the reciprocal of the dilution at which the average optical density (OD) at $450 \mathrm{~nm}$ value was greater than the mean of negative control sera samples plus 10 standard deviations (SD) of the mean or 0.1 , whichever was greater [17].

\section{Challenge assay}

Two weeks after the last dose of intranasal and oral immunizations, the remaining eight guinea pigs from each immunized and control groups were used for challenge experiment. The guinea pigs were conjunctively inoculated [18] with live $S$. dysenteriae type $4\left(20 \mu \mathrm{l}\right.$ each, $\sim 5 \times 10^{8} \mathrm{CFU} / \mathrm{ml}$, corresponding to five times of the infective dose 50). All these animals were observed daily for 5 days and scored for the development of disease or protection [12, 17].

One week after the second immunization dose, the remaining six guinea pigs from the intrarectally immunized group were inoculated with $10^{9} \mathrm{CFU}$ of the S. dysenteriae type 4 in $100 \mu \mathrm{lBS}$ through intrarectal route [14]. After $24 \mathrm{~h}$, two of the guinea pigs from each group were monitored for physiological and macroscopic changes, and the others were sacrificed to collect the distal region of the colon to examine the histopathological changes. Randomly selected distal regions of the colon were washed with PBS and fixed in $4 \%$ formaldehyde for $1 \mathrm{~h}$ at $4{ }^{\circ} \mathrm{C}$. The tissues were dehydrated by gradually soaking in alcohol and xylene and were embedded in paraffin [19]. Formalin-fixed, paraffin-embedded 
slides were sectioned at $6 \mu \mathrm{m}$ thickness, stained with hematoxylin and eosin, and examined under microscope. For evaluation of the biopsy specimens, histopathological features were selected as described by Rabbani et al. [20]. The criteria were as follows: (i) mucosal erosions were considered only when neutrophils or bleeding were present at the site of damage, (ii) cellular infiltrate within the lamina propria was subjectively assessed for increase in total number and relative number of neutrophils and mononuclear cells, and (iii) distorted crypt architecture was considered if there was crypt branching or when the regular and parallel array of crypts was deranged or irregular shape of crypt epithelial cells leading to damage of crypt architecture. Thus, histopathological changes were graded as mild, moderate, or severe. Normal histology or chronic inflammation was graded as 0 . Mild inflammation was diagnosed when the epithelial lining was intact with some cellular infiltrate and an increase in inflammatory cell in the lamina propria and with or without edema. Specimens with moderate changes had focal erosions of the epithelial surface and a pronounced increase in cellular infiltrate in the lamina propria and derangement of the crypts. Severe inflammation was diagnosed in presence of diffused mucosal erosion with surface exudates and damaged crypt architecture or crypt abscesses.

\section{Statistical analysis}

Mean \pm standard error of measurement or mean \pm SD were determined, and ELISA OD titers were compared using Student's $t$-test. A statistical comparison of protection data was determined using Fisher's exact test. $p$ values of $<0.05$ were considered significant.

\section{Results}

\section{Protective efficacy of the live DM104 in guinea pig model}

Guinea pigs, which were immunized through intranasal or oral route and later ocularly challenged 2 weeks after the last dose with wild-type $S$. dysenteriae type 4, were observed for the onset of symptoms [17]. Three of the eight intranasally immunized guinea pigs completely resisted keratoconjunctivitis and two guinea pigs showed mild keratoconjunctivitis after 3 days of challenge (Table I). The remaining three guinea pigs showed fully developed keratoconjunctivitis. In case of orally immunized group, two guinea pigs showed mild inflammation, whereas the remaining six guinea pigs showed fully developed keratoconjunctivitis with or without purulence. However, all 
Table I. Protective efficacy of the DM104 in guinea pig's eyes ${ }^{\mathrm{a}}$ immunized through intranasal or oral routes

\begin{tabular}{|c|c|c|c|c|c|c|c|}
\hline \multirow[b]{2}{*}{ Immunization with } & \multirow[b]{2}{*}{ Route of immunization } & \multicolumn{4}{|c|}{$\begin{array}{c}\text { No. of eyes } \\
(n=8) \text { with rating }\end{array}$} & \multirow[b]{2}{*}{ 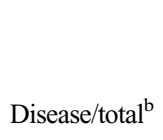 } & \multirow[b]{2}{*}{ Protection $(\%)$} \\
\hline & & 0 & 1 & 2 & 3 & & \\
\hline \multirow[t]{2}{*}{ PBS (control) } & Intranasal & 0 & 0 & 0 & 8 & $8 / 8$ & 0 \\
\hline & Oral & 0 & 0 & 1 & 7 & $8 / 8$ & 0 \\
\hline \multirow[t]{2}{*}{ DM104 } & Intranasal & 3 & 2 & 2 & 1 & $3 / 8$ & 63 \\
\hline & Oral & 0 & 2 & 5 & 1 & $6 / 8$ & 25 \\
\hline
\end{tabular}

${ }^{\text {a Guinea pigs were immunized intranasally or orally on days } 0,14 \text {, and } 28 \text { and later ocularly challenged on }}$ day 42 with $S$. dysenteriae 4 . Development of disease was rated as follows: 0 , no sign and symptom of inflammation; 1 , mild keratoconjunctivitis; 2 , keratoconjunctivitis without purulence; 3 , severe keratoconjunctivitis with purulence.

${ }^{\mathrm{b}}$ Guinea pigs were considered unhealthy if they had a rating of 2 or 3 .

${ }^{\mathrm{c}}$ Percentage of protection was calculated by the following formula:

$$
\left[\frac{\% \text { of disease in controls }-\% \text { of disease in vaccines }}{\% \text { of disease in controls }}\right] \times 100
$$

eight control guinea pigs immunized with PBS developed keratoconjunctivitis within 4 days of the live-cell challenge. If we consider the number of eyes with a rating of 2 or 3 versus eyes with a rating of 0 or 1 , then these results indicated $63 \%$ and $25 \%$ protective efficacy of the DM104 isolate in the intranasally and orally immunized guinea pig eye model, respectively.

After $24 \mathrm{~h}$ following challenge with $10^{9} \mathrm{CFU}$ of $S$. dysenteriae type 4 by the intrarectal route, guinea pigs immunized with PBS developed bacillary dysentery characterized by weight loss, tenesmus, and liquid stool mixed with mucus and blood (Table II). Furthermore, the body temperature increased by $\sim 2{ }^{\circ} \mathrm{C}$ at $24 \mathrm{~h}$ after challenge compared with that of DM104-immunized group. Macroscopically, the distal region of the large intestine of PBS-immunized guinea pigs only showed internal hemorrhage, whereas the group of guinea pigs immunized with the DM104 strain did not suffer from the abovementioned symptoms. To examine the histopathological changes, the distal regions of the colon in the guinea pigs were observed for tissue destruction and inflammation after $24 \mathrm{~h}$ following intrarectal challenge with $S$. dysenteriae type 4 . This examination revealed that the DM104-immunized group and the control group differed significantly $(p<0.05)$. Severe inflammation was observed in the distal colons of the control group guinea pigs receiving PBS (Figure 1a), whereas distal colons of the DM104immunized group appeared normal (Figure 1b) except for one of the guinea pigs that suffered from mild inflammation in the colon. The result of inflammation 
Table II. Evaluation of the DM104-immunized guinea pigs (through intrarectal route) for protection assays $^{\mathrm{a}}$

\begin{tabular}{|c|c|c|c|c|c|c|}
\hline \multirow[b]{2}{*}{$\begin{array}{l}\text { Immunization } \\
\text { with }\end{array}$} & \multirow[b]{2}{*}{$\begin{array}{l}\text { Challenge } \\
\text { with }\end{array}$} & \multicolumn{2}{|c|}{ Body weight (g) } & \multirow[b]{2}{*}{$\begin{array}{l}\text { Frequency } \\
\text { of tenesmus }\end{array}$} & \multirow{2}{*}{$\begin{array}{l}\text { Stool mixed } \\
\text { with mucus } \\
\text { and blood }\end{array}$} & \multirow{2}{*}{$\begin{array}{c}\text { Body } \\
\text { temperature } \\
\left({ }^{\circ} \mathrm{C}\right)\end{array}$} \\
\hline & & Initial & Terminal & & & \\
\hline PBS & $\begin{array}{l}\text { S. dysenteriae } \\
\text { type } 4\end{array}$ & $290 \pm 2$ & $250 \pm 5$ & $2-3$ & + & Increased \\
\hline DM104 & & $283 \pm 5$ & $290 \pm 2$ & 0 & - & No change \\
\hline
\end{tabular}

${ }^{\mathrm{a}}$ Guinea pigs were immunized through the intrarectal route with $5 \times 10^{8} \mathrm{CFU}$ of DM104 cells in $100 \mu \mathrm{PBS}$ on days 0 and 14. One week after the second immunization, both groups were challenged with $S$. dysenteriae type 4 . Physiological changes that included weight loss, tenesmus, change in body temperature, and stool were monitored at $24 \mathrm{~h}$ after challenge. PBS-immunized group was considered as negative control.
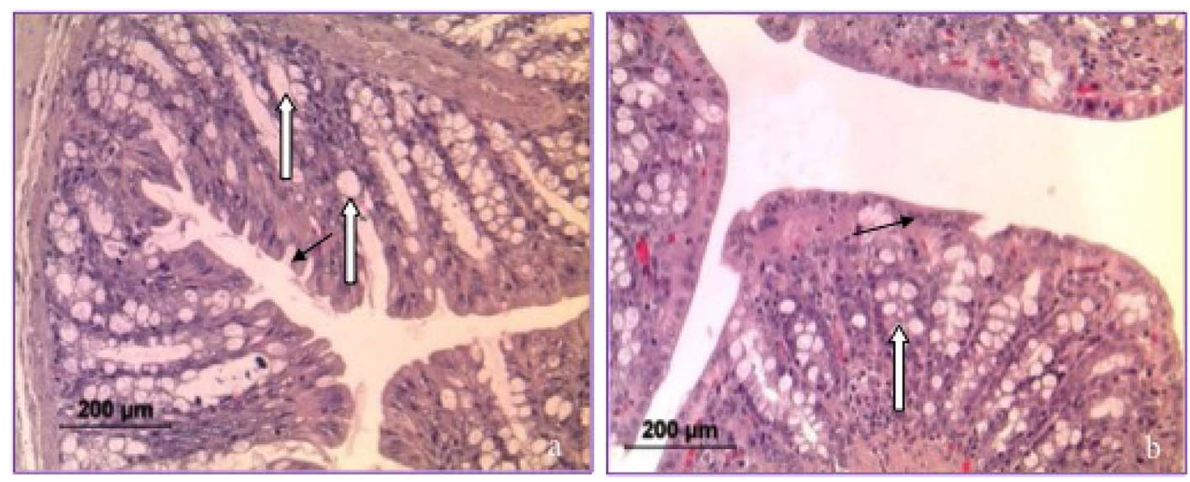

Figure 1. Histopathological features of the large intestine of the immunized guinea pigs following intrarectal challenge with $S$. dysenteriae type 4 . Samples of the distal colon were taken at $24 \mathrm{~h}$ after challenge. Distal colon of control guinea pigs showed degenerated epithelial line (arrow) and irregular-shaped crypt structure indicative of severe infection (block arrow) (a). DM104-immunized guinea pigs showed intact epithelial line (arrow) and regular- and parallel-shaped crypt structure

(block arrow) (b)

grade after challenge was found to be statistically significant between the immunized and control groups of guinea pigs $(p<0.05)$.

Immune response

Immunogenicity of the live DM104 was measured by determining the levels of serum $\operatorname{IgG}$ and $\operatorname{IgA}$ and mucosal $\operatorname{Ig} \mathrm{A}$ in eye wash and intestinal lavage fluid 


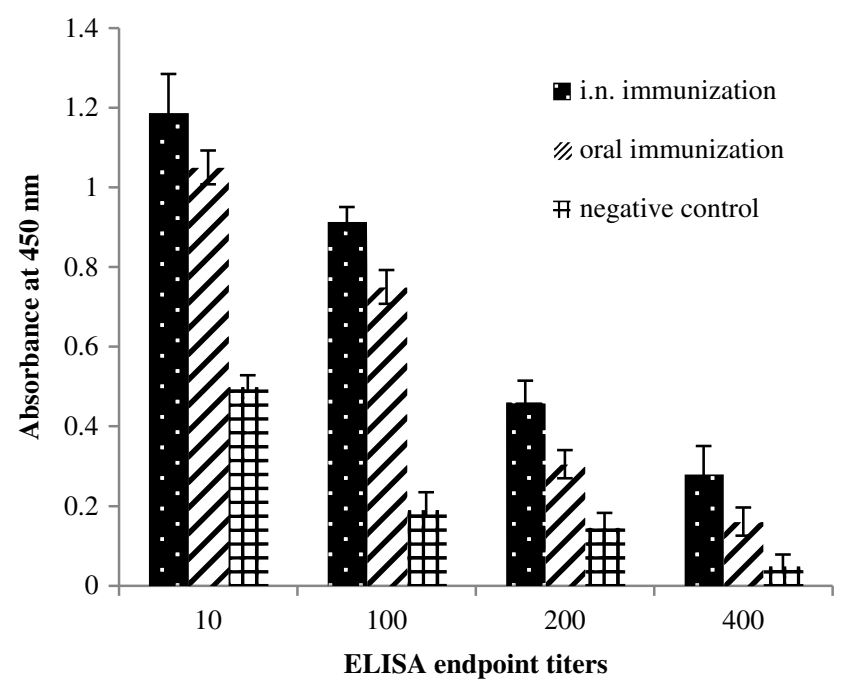

a. DM104 WCL-specific IgG

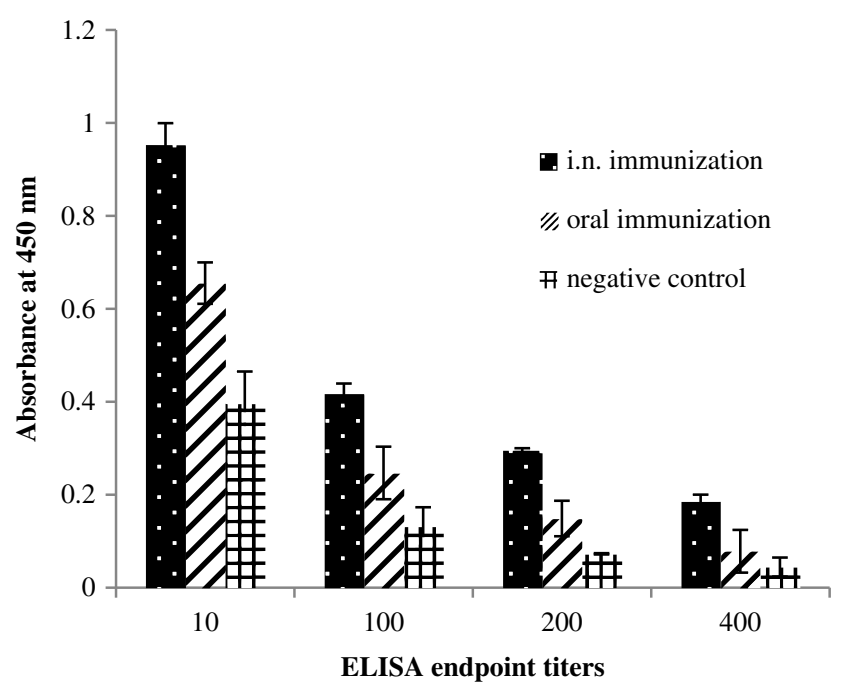

b. S. dysenteriae type 4 WCL-specific IgG

Figure 2. Serum IgG level against live DM104. Guinea pigs were immunized through intranasal (i.n.) or oral route with live DM104 on days 0,14 , and 28, and sera were collected 2 weeks after the last immunization before challenge. Serum IgG titers (mean \pm SD) against DM104 (a) and S. dysenteriae type 4 (b) WCL antigens from immunized guinea pigs were measured and found to be higher when compared with the control guinea pigs, administered with PBS only. Data represent the geometric mean titers for each group of guinea pigs immunized intranasally or orally with DM104. The titers were defined as the reciprocal of the last dilution having an optical density of 0.1 or more at $450 \mathrm{~nm}$ 
directed against DM104 and $S$. dysenteriae type 4 WCL by ELISA. The results shown in Figure 2 demonstrate significant differences in the levels of antibodies elicited by the two different immunization routes $(p<0.05)$. Higher levels of IgG were observed in animals immunized intranasally than in those immunized orally (Figure 2). Serum IgG titer against homologous DM104 WCL antigen was also found to be quite higher (Figure 2a) compared with the IgG titer against crossreactive $S$. dysenteriae type $4 \mathrm{WCL}$ antigen (Figure 2b). However, the serum geometric mean titer of the WCL-specific IgA was found to be significantly low. The IgA levels found in the mucosal secretions of eye wash and intestinal lavage fluid of the immunized guinea pigs were found to be quite significant against both the DM104 and S. dysenteriae type 4 WCL antigens (Figure 3). When considered as a group, the serum geometric mean titers of the intranasally immunized WCLspecific IgA (Figure 3) was found to be significantly higher when compared with the orally immunized WCL-specific IgA (Figure 3$)(p<0.05)$. However, serum $\mathrm{IgG}$ and $\mathrm{IgA}$ titers in intrarectally immunized guinea pigs were found to be very low (data not shown).

\section{Discussion}

In this study, immunogenicity was evaluated by assessing the ability of the E. albertii strain DM104 to protect guinea pigs immunized through either intranasal, oral, or intrarectal route against challenge with $S$. dysenteriae type 4. We addressed how the route of immunization influenced the quality of the immune response with regard to protection against challenge. Our results indicate that the immune response elicited by vaccine candidate strain DM104 is strongly dependent on the immunization route, with the intranasal route being more efficient than the oral route following conjunctival challenge. Immunization yielded $63 \%$ and $25 \%$ protective efficacy in intranasal- and oral-immunized guinea pigs, respectively, against development of keratoconjunctivitis. Protection generated by intranasal immunization was accompanied by highly specific anti-WCL IgA titers in eye wash and intestinal lavage fluid of DM104-immunized guinea pigs. These experiments are consistent with the previous studies that reported using the intranasal model of guinea pig vaccination, that high titers of anti-LPS IgA were associated with protection [21-23]. Moreover, in the guinea pig keratoconjunctivitis model, high levels of antigen-specific antibody-secreting cells occur in superficial ventral cervical lymph nodes and correlate with the protective efficacy of a vaccine [24].

Consistent with other studies [25] that focused on the differences between the immune response following either intranasal or oral antigen administration, we also found that higher titers of serum anti-WCL IgG were observed in guinea pigs 


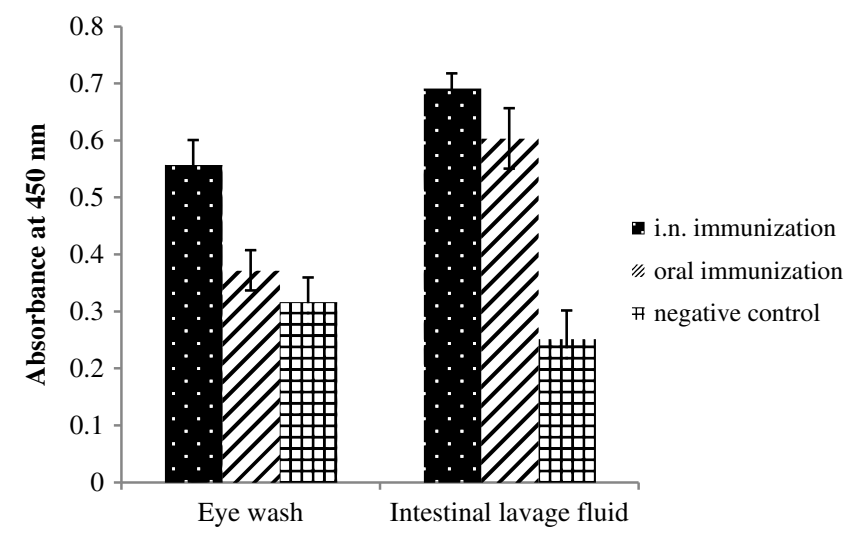

a. DM104 WCL-specific IgA

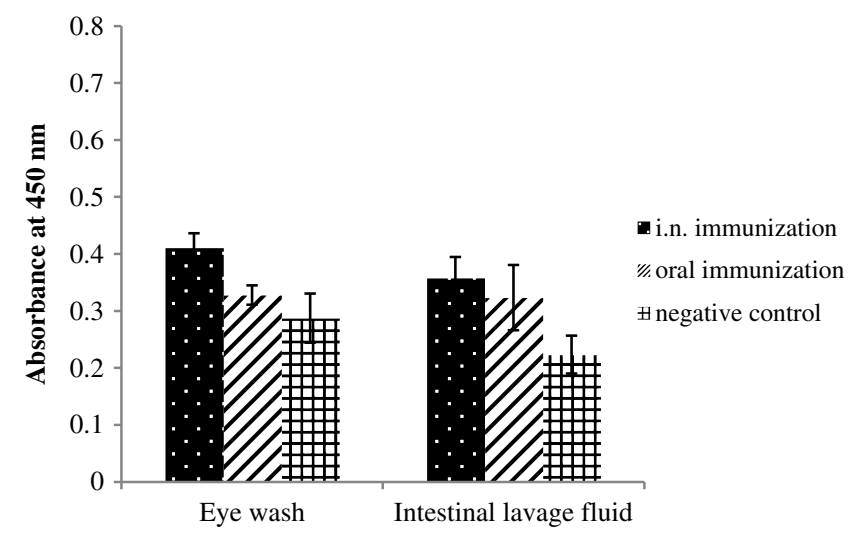

b. S. dysenteriae type 4 WCL-specific IgA

Figure 3. Mucosal IgA level against live DM104. Guinea pigs were immunized through intranasal (i.n.) or oral route with live DM104 on days 0,14 , and 28, and mucosal secretions were collected 2 weeks after the last immunization before challenge. Mucosal IgA level (mean \pm SD) in eye wash and intestinal lavage fluid against WCL antigen of DM104 (a) and S. dysenteriae type 4 (b) from immunized guinea pigs were measured and found to be higher when compared with the control guinea pigs, administered with PBS only

immunized intranasally than in those immunized orally with DM104. However, it has been shown that in the murine pulmonary model of shigellosis, there was no correlation between high anti-LPS IgG and protection [26]. However, in this study, under both the intranasal-oral immunizations, DM104 stimulated significant levels of anti-WCL IgG, suggesting that these antibodies might have influenced the protection in the guinea pig model. 
The intranasal route of administration is becoming increasingly popular in experimental animal models to elicit an immune response either in the airway mucosa or in the distal mucosal sites. Studies with diverse antigens in various animal species have demonstrated that the intranasal route of administration can elicit a broad immune response, including serum, salivary, nasal, rectal, and vaginal antibodies, that is often superior to responses obtained after oral immunizations $[15,27,28]$.

On the other hand, Shim et al. [14] established a new bacillary dysentery model in the guinea pig by inoculating Shigella through the intrarectal route. An intrarectal challenge with wild-type strains of $S$. flexneri type $2 \mathrm{a}$ or $5 \mathrm{a}$ induced severe and acute bacillary dysentery that mimicked human shigellosis in terms of weight loss, fever, tenesmus, severe damage to the colonic mucosa, enhanced expression of proinflammatory cytokines such as IL-8, and predominant recruitment of neutrophils. This novel model was followed and reproduced in this study when results of the intrarectal model experiment also demonstrated the safety and protective efficacy of the live non-invasive Shigella-cross-reacting strain DM104 against $S$. dysenteriae type 4 infections.

A previous study using rectal biopsy specimens from acute Shigella dysentery patients identified the histopathological symptoms that are characteristic for human bacillary dysentery [29]. The majority of shigellosis patients show marked damage of the epithelial barrier, cellular infiltration (lymphocytes, plasma cells, and neutrophils), dramatic tissue injury (erosion, crypt alterations, necrosis, and edema), goblet cell depletion, and watery diarrhea [29-31]. General histological studies of rectal biopsies of Shigella-infected humans revealed epithelial hyperplasia, tissue destruction, and penetration of crypts into the submucosa [29]. Shim et al. [14] found the same symptoms and histology in this guinea pig model, confirming that it successfully mimics shigellosis symptoms in humans.

In another study, the immunogenicity and protective efficacy of a S. flexneri 2a vaccine candidate were compared with a live-attenuated Shigella vaccine strain following intranasal or ocular immunization of guinea pigs [17]. Similarly, this study, along with our previous study [12] carried out with $S$. dysenteriae type 4 vaccine strain DM104, clearly showed the protective efficacy of a vaccine in the guinea pig model. The high protection in guinea pig models induced by the DM104 strain along with its non-pathogenic properties strongly suggests that the Shigella-cross-reacting DM104 strain may be used as a promising live vaccine candidate against shigellosis. However, the mechanism of the immune response of DM104 in protection against live-cell challenge in different animal models remains to be done in future studies. 


\section{Acknowledgement}

This work was supported by The Norwegian Program for Development, Research and Higher Education (NUFU) (Grant nos. PRO 52/03 and 2007/10063).

\section{Conflict of Interest}

The authors declare no conflict of interest.

\section{References}

1. Black, R. E., Cousens, S., Johnson, H. L., Lawn, J. E., Rudan, I., Bassani, D. G., Jha, P., Campbell, H., Walker, C. F., Cibulskis, R., Eisele, T., Liu, L., Mathers, C.: Global, regional, and national causes of child mortality in 2008: A systematic analysis. Lancet 375, 1969-1987 (2010).

2. Kotloff, K. L., Winickoff, J. P., Ivanoff, B., Clemens, J. D., Swerdlow, D. L., Sansonetti, P. J., Adak, G. K., Levine, M. M.: Global burden of Shigella infections: Implications for vaccine development and implementation of control strategies. Bull World Health Organ 77, 651-666 (1999).

3. Peng, X., Luo, W., Zhang, J., Wang, S., Lin, S.: Rapid detection of Shigella species in environmental sewage by an immunocapture PCR with universal primers. Appl Environ Microbiol 68, 2580-2583 (2002).

4. Abo-Amer, A. E., Soltan, E. S. M., Abu-Gharbia, M. A.: Molecular approach and bacterial quality of drinking water of urban and rural communities in Egypt. Acta Microbiol Immunol Hung 55, 311-326 (2008).

5. Jennison, A. V., Verma, N. K.: Shigella flexneri infection: Pathogenesis and vaccine development. FEMS Microbiol Rev 28, 43-58 (2004).

6. Mukhopadhaya, A., Mahalanabis, D., Chakrabarti, M. K.: Role of Shigella flexneri 2a 34 $\mathrm{kDa}$ outer membrane protein in induction of protective immune response. Vaccine $\mathbf{2 4}$, 6028-6036 (2006).

7. Levine, M. M., Kotloff, K. L., Barry, E. M., Pasetti, M. F., Sztein, M. B.: Clinical trials of Shigella vaccines: Two steps forward and one step back on a long, hard road. Nat Rev Microbiol 5, 540-553 (2007).

8. Rahman, M. Z., Sultana, M., Khan, S. I., Birkeland, N. K.: Serological cross-reactivity of environmental isolates of Enterobacter, Escherichia, Stenotrophomonas, and Aerococcus with Shigella spp.-specific antisera. Curr Microbiol 54, 63-67 (2007).

9. Azmuda, N., Rahman, M. Z., Sultana, M., Jenssen, E. L., Khan, S. I., Birkeland, N. K.: Evidence of interspecies O antigen gene cluster transfer between Shigella boydii 15 and Escherichia fergusonii. APMIS 120, 959-966 (2012).

10. Rahman, M. Z., Akter, S., Azmuda, N., Sultana, M., Weill, F. X., Khan, S. I., Grimont, P. A., Birkeland, N. K.: Serological cross-reaction between O-antigens of Shigella 
dysenteriae type 4 and an environmental Escherichia albertii isolate. Curr Microbiol 67, 590-595 (2013).

11. Rahman, M. Z., Azmuda, N., Hossain, M. J., Sultana, M., Khan, S. I., Birkeland, N. K.: Recovery and characterization of environmental variants of Shigella flexneri from surface water in Bangladesh. Curr Microbiol 63, 372-376 (2011).

12. Chowdhury, F. M., Rahman, M. Z., Khan, S. I., Ahsan, C. R., Birkeland, N. K.: An environmental Escherichia albertii strain, DM104, induces protective immunity to Shigella dysenteriae in guinea pig eye model. Curr Microbiol 68, 642-647 (2014).

13. Bernardini, M. L., Arondel, J., Martini, I., Aidara, A., Sansonetti, P. J.: Parameters underlying successful protection with live attenuated mutants in experimental shigellosis. Infect Immun 69, 1072-1083 (2001).

14. Shim, D. H., Suzuki, T., Chang, S. Y., Park, S. M., Sansonetti, P. J., Sasakawa, C., Kweon, M. N.: New animal model of shigellosis in the guinea pig: Its usefulness for protective efficacy studies. J Immunol 178, 2476-2482 (2007).

15. Orr, N., Robin, G., Cohen, D., Arnon, R., Lowell, G. H.: Immunogenicity and efficacy of oral or intranasal Shigella flexneri 2a and Shigella sonnei proteosome-lipopolysaccharide vaccines in animal models. Infect Immun 61, 2390-2395 (1993).

16. Mel, D. M., Arsic, B. L., Nikolic, B. D., Radovanic, M. L.: Studies on vaccination against bacillary dysentery. 4 . Oral immunization with live monotypic and combined vaccines. Bull World Health Organ 39, 375-380 (1968).

17. Ranallo, R. T., Thakkar, S., Chen, Q., Venkatesan, M. M.: Immunogenicity and characterization of WRSF2G11: A second generation live attenuated Shigella flexneri 2a vaccine strain. Vaccine 25, 2269-2278 (2007).

18. Sereny, B.: Experimental keratoconjunctivitis shigellosa. Acta Microbiol Acad Sci Hung 2, 367-376 (1957).

19. Kweon, M. N., Yamamoto, M., Rennert, P. D., Park, E. J., Lee, A. Y., Chang, S. Y., Hiroi, T., Nanno, M., Kiyono, H.: Prenatal blockage of lymphotoxin $\beta$ receptor and TNF receptor p55 signaling cascade resulted in the acceleration of tissue genesis for isolated lymphoid follicles in the large intestine. J Immunol 174, 4365-4372 (2005).

20. Rabbani, G. H., Albert, M. J., Rahman, A. S. H., Islam, M. M., Islam, K. M. N., Alam, K.: Short-chain fatty acids improve clinical, pathologic, and microbiologic features of experimental shigellosis. J Infect Dis 179, 390-397 (1999).

21. Noriega, F. R., Losonsky, G., Lauderbaugh, C., Liao, F. M., Wang, J. Y., Levine, M. M.: Engineered $\Delta$ guaB-A $\Delta$ virG Shigella flexneri 2a strain CVD 1205: Construction, safety, immunogenicity, and potential efficacy as a mucosal vaccine. Infect Immun 64, 3055-3061 (1996).

22. Noriega, F. R., Losonsky, G., Wang, J. Y., Formal, S. B., Levine, M. M.: Further characterization of $\Delta$ aroA $\Delta$ virG Shigella flexneri 2a strain CVD 1203 as a mucosal Shigella vaccine and as a live-vector vaccine for delivering antigens of enterotoxigenic Escherichia coli. Infect Immun 64, 23-27 (1996).

23. Noriega, F. R., Liao, F. M., Maneval, D. R., Ren, S., Formal, S. B., Levine, M. M.: Strategy for cross-protection among Shigella flexneri serotypes. Infect Immun 67, 782-788 (1999).

24. Hartman, A. B., Vande Verg, L. L., Collins, H. H., Jr., Tang, D. B., Bendiuk, N. O., Taylor, D. N., Powell, C. J.: Local immune response and protection in the guinea pig keratoconjunctivitis model following immunization with Shigella vaccines. Infect Immun 62, 412-420 (1994). 
25. Wu, H. Y., Nikolova, E. B., Beagley, K. W., Eldridge, J. H., Russell, M. W.: Development of antibody-secreting cells and antigen-specific $\mathrm{T}$ cells in cervical lymph nodes after intranasal immunization. Infect Immun 65, 227-235 (1997).

26. Mallett, C. P., Vande Verg, L. L., Collins, H. H., Hale, T. L.: Evaluation of Shigella vaccine safety and efficacy in an intranasally challenged mouse model. Vaccine 11, 190-196 (1993).

27. Langermann, S., Palaszynski, S., Sadziene, A., Stover, C. K., Koenig, S.: Systemic and mucosal immunity induced by BCG vector expressing outer-surface protein A of Borrelia burgdorferi. Nature 372, 552-555 (1994).

28. Natuk, R. J., Davis, A. R., Chanda, P. K., Lubeck, M. D., Chengalvala, M., Murthy, S. C., Wade, M. S., Dheer, S. K., Bhat, B. M., Murthy, K. K.: Adenovirus vectored vaccines. Dev Biol Stand 82, 71-77 (1994).

29. Anand, B. S., Malhotra, V., Bhattacharya, S. K., Datta, P., Datta, D., Sen, D., Bhattacharya, M. K., Mukherjee, P. P., Pal, S. C.: Rectal histology in acute bacillary dysentery. Gastroenterology 90, 654-660 (1986).

30. Islam, M. M., Azad, A. K., Bardhan, P. K., Raqib, R., Islam, D.: Pathology of shigellosis and its complications. Histopathology 24, 65-71 (1994).

31. Islam, D., Veress, B., Bardhan, P. K., Lindberg, A. A., Christensson, B.: In situ characterization of inflammatory responses in the rectal mucosae of patients with shigellosis. Infect Immun 65, 739-749 (1997). 